\title{
Polarization Sensitive Optical Imaging and Characterization of Soybean Using Stokes- Mueller Matrix Model
}

\author{
Shamaraz Firdous \\ National Institute of Lasers E Optronics (NILOP), Islamabad, \\ Pakistan
}

\section{Introduction}

Light polarimetry is a useful tool by which to analyze the modification in the shape and orientation of the field vectors of the electromagnetic radiation which propagates through scattering medium. Among the methods available to analyze turbid media, the use of polarized light has attracted much attention recently, as it has been discovered that multiply scattered photons still maintain partial polarization. [1-4] A typical experiment entails launching a known polarization state in light into a turbid sample and measuring the polarization properties of the reemitted light. The detected signal depends on many variables, including the number and nature of scattering events, the incident polarization state, and the detection geometry. [5-7] In the past few years, several groups have shown how polarization sensitive scattering measurements can be used to measure certain properties of turbid medium such as the average particle size, [8] scattering coefficient, anisotropy factor of particle suspensions , [9] optical material characterization, [10-11] and the study of biological materials. [11-13] An optical polarizers and retarders are rotated to provide additional incident and analyzed polarization states to enable the reconstruction of the 2-D Mueller matrix of various biological sample [14-15]. It has also been shown that the benefits of using polarized light can be combined with different optical modalities. For example, the benefit of using of polarized light in optical coherence tomography (OCT) measurements can significantly improve image contrast. [16-17]

Furthermore, the measurement of polarization parameters of the light scattered benefits from a relatively simple, fast, and convenient data acquisition procedure, [18-19] which motivates the ongoing efforts aimed at further developing the scattering polarization imaging technology. If some of the light retained its polarization properties upon multiple scattering at $180^{\circ}$ transmittion mode and this effect could be quantified and exploited, potentially useful measurements could be made in almost any clinical situation. Since light in the visible and infrared regions of the electromagnetic spectrum is not harmful to biological tissues at moderate flounce levels, has a penetration depth of several millimeters, and has a reasonable chance of scattering out of the tissue and being detected, it would be ideal for making noninvasive measurements. Other practical reasons for studying the behavior of light at $180^{\circ}$ would be for the possibility of spatial imaging to map out the locations of sample structures and compositions, and to gain a better general understanding of turbid systems. [20-22] 
A comprehensive understanding of light propagation and scattering for the most general case of highly scattering media is yet to be attained. An analysis based on the Stokes vector and Mueller matrix approach provides a theoretical framework, which can be directly related to the experimentally measurable parameters [23-24]. The Stokes vector - Mueller matrix approach for scattering has been extended to characterization of spatially varying polarization patterns for scattered light. In this approach, determining 16 components of the Mueller matrix for the studied object gives a comprehensive description of scattering properties of a sample or a medium in the spatial domain. Rather then being just one number, each of the 16 components of the Mueller matrix is, in fact, a two-dimensional (2D) array of numbers, corresponding to different spatial locations across the surface of the object or medium.[25]

In this study, we consider experimental Mueller matrix of soybean oil (highly tissue like phantom) for their polarization and depolarization observations. The transmitted photons preserve their polarization memory and Mueller matrix represents this information in the form of matrix array and intensity patterns.

\section{Optical properties of the scatterer that influence polarization}

The scattering through soybean oil is the principle mechanism that modifies the initial polarization state of the incident light. The polarization state of light after a single scattering event depends on the direction of scattering and incident polarization state. [26-27] In many turbid media such as tissue, scattering structures have a large variance in size and are distributed or oriented in a complex and sometimes apparently random manner. Because each scattering event can modify the incident polarization state differently, until finally the polarization state is completely randomized. An important exception is when the media consists of organized linear structures, such as birefrengent soybean oil, and then the phase retardation between orthogonal polarization components is proportional to the distance traveled through the birefrengent medium. The phase retardation of the scattering medium is given as.

$$
\delta=\frac{2 \pi \Delta n x}{\lambda}
$$

The phase retardation measurement through turbid media is aimed at retrieving useful information from such multiply scattered light. The behavior of light in random media is well-known from the extensive study of wave propagation. Light traveling in a random medium can be classified into three categories, the ballistic, the snake and the diffuse light. The ballistic light either remains unscattered, or undergoes coherent forward scattering in the medium. This light travels undeviated and has the shortest path length in the medium. The snake light is that which undergoes near-forward scattering, and follows path that undulate about the ballistic path.[28] The diffuse photons largely exceed the other two categories in number and undergoes multiple scattering. We considered all three kinds of photons in our study.

A large number of different experiments are possible if one wants to study the polarization dependent scattering properties of turbid media. The probing light may be linearly polarized at various angles, right and left-hand circularly, or elliptically polarized. Light coming from the scattering medium can be analyzed in the same numerous ways. However, only a few measurements are needed to completely characterize the optical properties of 
any material. The necessary procedure is elegantly demonstrated by the Stokes-vector Mueller matrix approach to polarization and light scattering.

\section{Stokes vector-Mueller matrix formulism}

The research of polarized scattered light deals with the entire scattering process in the context of Stokes-Mueller matrices and polarizations. [29] An introduction to optical polarization often starts with a description of the optical elements which physically act as polarizers and retarders. The Stokes vector-Mueller matrix- calculus is then used to show mathematically how these optical elements affect a light beam.

A Stokes vector, a $4 \times 1$ vector, is a mathematical representation of the polarization state of light. [30] It can be represented as a set of six intensity measurements recorded through a set of various polarizing filters. The Stokes vector is composed of four elements, $I, Q, U$, and $V$ and provides a complete description of the light polarization state. If the total irradiant intensity $I_{t}$ incident on the sample and $I_{00}, I_{90}{ }, I_{+45}, I_{-45}, I_{r c}$, and $I_{l c}$ the irradiances transmitted by a polarizer-retarders are focused to the detector, then, the Stokes parameters are defined by:[30]

$$
S=\left[\begin{array}{c}
I \\
Q \\
U \\
V
\end{array}\right]=\left[\begin{array}{c}
S_{0} \\
S_{1} \\
S_{2} \\
S_{3}
\end{array}\right]=\left[\begin{array}{c}
I_{t} \\
I_{0}-I_{90} 0 \\
I_{{ }^{445} 0}-I_{-450} 0 \\
I_{r c}-I_{l c}
\end{array}\right]=\left[\begin{array}{l}
\left\langle E_{0 x}^{2}\right\rangle+\left\langle E_{0 y}^{2}\right\rangle \\
\left\langle E_{0 x}^{2}\right\rangle-\left\langle E_{0 y}^{2}\right\rangle \\
2\left\langle E_{0 x} E_{0 y} \cos \delta\right\rangle \\
2\left\langle E_{0 x} E_{0 y} \sin \delta\right\rangle
\end{array}\right]
$$

\begin{tabular}{|c|c|c|c|}
\hline $\mathbf{S}_{11}($ Parallel x Parallel) & $\begin{array}{l}\mathbf{S}_{12} \text { (horizontal } \\
\text { Parallel) }\end{array}$ & $\mathbf{S}_{13}\left(45^{\circ} \times\right.$ Parallel $)$ & $\begin{array}{l}\mathbf{S}_{14} \\
\text { Parallel ) }\end{array}$ \\
\hline $\mathrm{I}_{00}$ & $\mathrm{In}_{0}-\mathrm{I}_{\mathrm{v}}$ & $I_{+0}+t_{0}$ & $\mathrm{~L}_{L^{-}} \mathrm{I}_{\mathrm{R} 0}$ \\
\hline $\begin{array}{l}\mathbf{S}_{21} \quad \text { (Parallel } \\
\text { horizontal) }\end{array}$ & $\begin{array}{ll}\mathbf{S}_{22} & \text { (horizontalx } \\
\text { horizontal) } & \end{array}$ & $\begin{array}{l}S_{23} \\
\text { horizontal) }\end{array}$ & $\begin{array}{l}\mathrm{S}_{24} \\
\text { horizontal) }\end{array}$ \\
\hline $\mathrm{I}_{\mathrm{on}}-\mathrm{I}_{0 \mathrm{v}}$ & $\left(I_{\mathrm{hn}}+\mathrm{I}_{\mathrm{vv}}\right)-\left(\mathrm{I}_{\mathrm{vh}}+\mathrm{I}_{\mathrm{hv}}\right)$ & $\left(I_{+h}+I_{-v}\right)-\left(I_{-h}+I_{+v}\right)$ & $\left(I_{L n}+I_{R v}\right)-\left(I_{R h}+I_{L v}\right)$ \\
\hline $\mathrm{S}_{31}\left(\right.$ Parallel $\left.\times 45^{\circ}\right)$ & $\mathrm{S}_{32}\left(\right.$ horizontal $\left.\mathrm{x} 45^{\circ}\right)$ & $S_{33}\left(45^{0} \times 45^{\circ}\right)$ & $\mathrm{S}_{34}\left(\right.$ L.circularx $\left.45^{\circ}\right)$ \\
\hline$\left(I_{0}+I_{0 .}\right)$ & $\left(I_{n_{+}}+I_{v_{-}}\right)-\left(I_{v_{+}}+I_{n_{-}}\right)$ & $\left(I_{++}+1_{+-\ldots}\right)-\left(I_{-+}+1_{+}\right)$ & $\left(I_{L}+I_{R_{-}}\right)-\left(I_{R_{+}}+I_{L_{-}}\right)$ \\
\hline $\begin{array}{l}\mathbf{S}_{41} \quad \text { (Parallel } \\
\text { L.circular) }\end{array}$ & $\begin{array}{l}\mathbf{S}_{42} \text { (horizontal } \\
\text { L.circular) }\end{array}$ & $\mathbf{S}_{43}\left(45^{\circ} \times\right.$ L.circular $)$ & $\begin{array}{l}S_{44}(\text { L.circularxR.circul } \\
\text { er) }\end{array}$ \\
\hline$\left(\mathrm{I}_{\mathrm{OL}}-\mathrm{I}_{\mathrm{OR}}\right)$ & $\left(I_{\mathrm{hL}}+\mathrm{I}_{\mathrm{VR}}\right)-\left(\mathrm{I}_{\mathrm{vL}}+\mathrm{I}_{\mathrm{hR}}\right)$ & $\left(I_{+L}+I_{-R}\right)-\left(I_{-L}+I_{+R}\right)$ & $\left(I_{L L}+I_{R R}\right)-\left(I_{R L}+I_{L R}\right)$ \\
\hline
\end{tabular}

Table 1. A matrix array showing the polarization measurements, necessary to measure each particular matrix element of the different configurations (polarizer and analyzer) setup. 
Where Ex and Ey are the electric field vectors along $x$ and $y$ direction and $\delta$ is angle. After normalizing the Stokes parameters by the irradiance $I, Q$ describes the amount of light polarized along the horizontal $(Q=+1)$ or vertical $(Q=-1)$ axes, $U$ describes the amount of light polarized along the $+45^{\circ}(U=+1)$ or $-45^{\circ}(U=-1)$ directions, and $\mathrm{V}$ describes the amount of right $(V=+1)$ or left $(V=-1)$ circularly polarized light.

A Mueller matrix, a $4 \times 4$ matrix, is a mathematical description of how an optical sample interacts or transforms the polarization state of an incident light beam and given as [30]

$$
[M]=\left[\begin{array}{llll}
m_{11} & m_{12} & m_{13} & m_{14} \\
m_{21} & m_{22} & m_{23} & m_{24} \\
m_{31} & m_{32} & m_{33} & m_{34} \\
m_{41} & m_{42} & m_{43} & m_{44}
\end{array}\right]
$$

where, $\mathrm{M}$ is the $4 \times 4$ Mueller matrix of the media or sample and can be experimentally measured through the application of various incident polarization states and then by analyzing the state of polarization of the light leaving the sample. Since a Mueller matrix contains 16 elements (mij) of the matrix $\mathrm{M}$ and reconstruction requires 49 independent polarization measurements according to different polarizer and wave plate orientation as shown in table.1 and Fig.1. [31] The Mueller matrix can be thought of as the "optical fingerprint" of a sample. This matrix operates directly on an input or incident Stokes vector, thus resulting in an output $4 \times 1$ Stokes vector that describes the polarization state of the light leaving the sample. This is described mathematically by the equation given as [32]

$$
\left[S_{\text {out }}\right]=\left[M_{\text {system }}\right]\left[S_{\text {in }}\right]
$$

where $\left[S_{\text {out }}\right]$ the output Stokes vector, $\left[S_{\text {in }}\right]$ the Stokes input vector and $\left[M_{\text {system }}\right]$ is the Mueller matrix representing the entire experimental optical system given as

$$
\left[M_{\text {system }}\right]=[Q W]\left[A_{M}\right][M][Q W]\left[P_{M}\right]
$$

The output stokes vector $\left[S_{\text {out }}\right]$ can be calculated by relation in Eq. 4, putting the values of the Mueller matrix for optics and the Mueller matrix of the system. [33] The complete characterization of the polarization state of light by means of the Stokes parameters permits the calculation of the degree of polarization (DOP), defined as

$$
D O P=\sqrt{\frac{Q^{2}+U^{2}+V^{2}}{I}}=\sqrt{\frac{S_{1}{ }^{2}+S_{2}{ }^{2}+S_{3}{ }^{2}}{S_{0}{ }^{2}}}
$$

For purely polarized light, the degree of polarization is unity i.e. 1, and the Stokes parameters obey the equality $\mathrm{I}^{2}=\mathrm{Q}^{2}+\mathrm{U}^{2}+\mathrm{V}^{2}$, while for partially polarized light, the degree of polarization is smaller than unity, leading to $\mathrm{I}^{2}>\mathrm{Q}^{2}+\mathrm{U}^{2}+\mathrm{V}^{2}$. An input beam can be decomposed into purely polarized beams. After propagation through an optical system, the Stokes parameters of the purely polarized beam components are added to give the Stokes parameters for the original input beam. [34] 


\section{Determining the Mueller matrix}

If the Mueller matrix is not known, all the elements can be determined experimentally. It can be shown that 49 intensity measurements with various orientations of polarizers and analyzers are necessary to obtain the 16 elements of the Mueller matrix. [25]

$$
\begin{aligned}
& m_{11}=I_{00} \\
& m_{12}=I_{h 0}-I_{v 0} \\
& m_{13}=I_{+0}-I_{-0} \\
& m_{14}=I_{l 0}-I_{r 0} \\
& m_{21}=I_{0 h}-I_{0 v} \\
& m_{22}=\left(I_{h h}+I_{v v}\right)-\left(I_{v h}+I_{h v}\right) \\
& m_{23}=\left(I_{+h}+I_{-v}\right)-\left(I_{-h}+I_{+v}\right) \\
& m_{24}=\left(I_{l h}+I_{r v}\right)-\left(I_{r h}+I_{l v}\right) \\
& m_{31}=I_{0+}+I_{0-} \\
& m_{32}=\left(I_{h+}+I_{v-}\right)-\left(I_{v+}+I_{h-}\right) \\
& m_{33}=\left(I_{++}+I_{--}\right)-\left(I_{-+}+I_{+-}\right) \\
& m_{34}=\left(I_{l+}+I_{r-}\right)-\left(I_{r+}+I_{l-}\right) \\
& m_{41}=I_{0 l}-I_{0 r} \\
& m_{42}=\left(I_{h l}+I_{v r}\right)-\left(I_{v l}+I_{h r}\right) \\
& m_{43}=\left(I_{+l}+I_{-r}\right)-\left(I_{-l}+I_{+r}\right) \\
& m_{44}=\left(I_{l l}+I_{r r}\right)-\left(I_{r l}+I_{l r}\right)
\end{aligned}
$$

Where the first term represents the input polarization state while the second the output polarization state of light. The states are defined as: $\mathrm{h}=$ horizontal, $\mathrm{v}=$ vertical $,+=+45^{\circ},-=$ $-45^{\circ}, \mathrm{r}=$ right circular and $1=$ left circular. Once all 16 elements of the matrix are obtained, the medium is completely described in terms of its optical properties.

\section{Error analysis of Mueller matrix polarimeter}

For the retardations close to $0^{0}$ or $90^{0}$ the background noise on the detectors introduces a significant and systematic error of $15^{\circ}$ at a signal to noise ratio of $10 \mathrm{~dB}$. [35] The coherent detection scheme which calculates the Stokes parameters has better immunity to the system. in the calculation of the $Q$ parameter the spectral density in one polarization channel is subtracted from the spectral density in the orthogonal polarization channel, thus eliminating constant background noise terms, and the $U$ and $V$ parameters are calculated from the cross correlation between the orthogonally polarized channels, eliminating autocorrelation noise. Noise will decrease the degree of polarization, since it will be present as autocorrelation noise in the Stokes parameter I. In the incoherent detection scheme only $V$ is measured and the error in the phase retardation is introduced by the decrease of the amplitude of oscillations with increasing depth. In the coherent detection scheme, the Stokes parameters $Q, U$, and $\mathrm{V}$ can be renormalized on DOP, restoring the amplitude of the oscillations, and thus eliminating the systematic error.

We have analyzed system errors introduced by the extinction ratio of polarizing optics and chromatic dependence of wave retarders, and errors due to dichroism, i.e., the differences in 
the absorption and scattering coefficients for polarized light in soybean oil. System errors can be kept small by careful design of the system with achromatic elements, but can never be completely eliminated. Dichroism is a more serious problem when interpreting the results as solely due to birefringence. However, Mueller matrix polarimetry measurements have shown that the error due to dichroism is relatively small. [36] The variance in the computed Stokes vectors of transmitted light (excluding effect of birefringence) is due to multiple scattering, speckles, and shot noise (i.e., optimized system). At some depth, the detected signals are limited by shot noise. At shallower depths (i.e., before the shot noise limit) variance in the Stokes parameters is primarily due to the effects of multiple scattering and speckle. Multiple scattering scramble the polarization mainly in a random manner and this offers some means to distinguish it from birefringence. Thus, birefringence induced changes are relatively slow, and the Stokes parameters change according to the Mueller matrix of a linear retarder. [37] However, an optic axis that varies with depth will give changes in the polarization state that will be difficult to distinguish from the random manner of multiple scattering. More research is necessary on this complex problem. We use coherent light source and standard optical filters to minimize these errors for our Mueller matrix polarimeter.

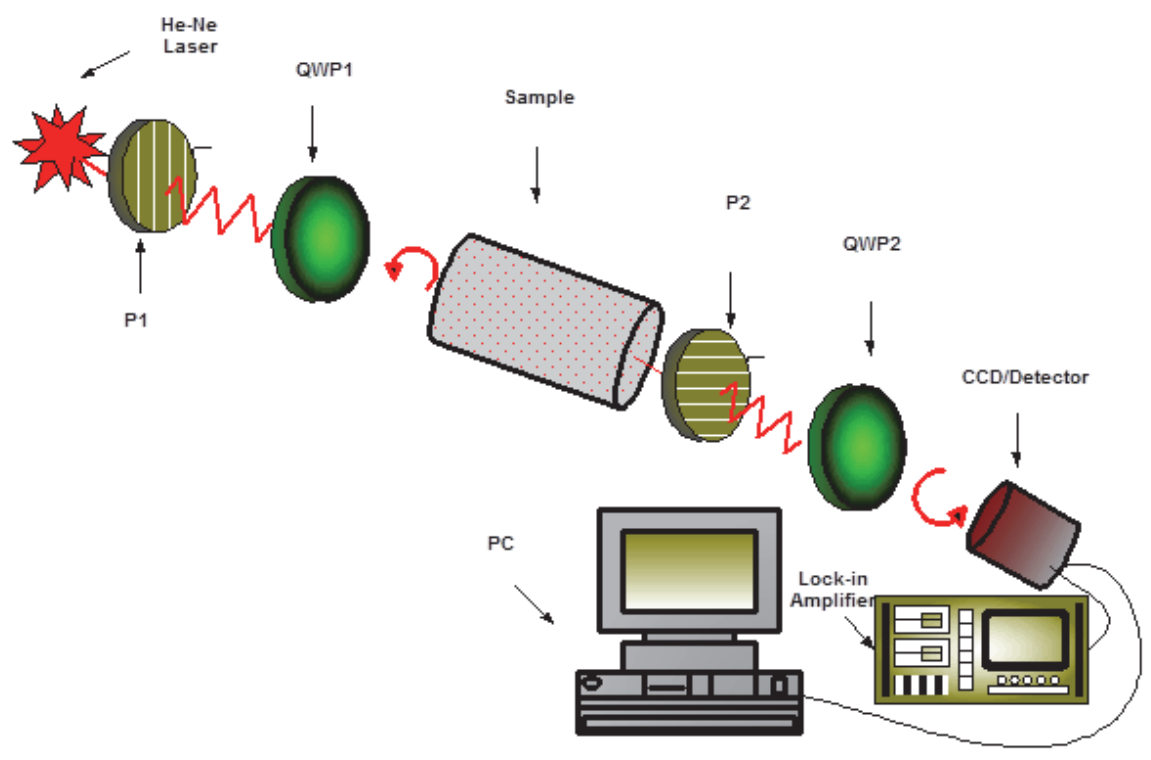

Fig. 1. Experimental setup for measurements of transmitted Mueller matrix elements. A HeNe laser beam with an output power of $5 \mathrm{~mW}$ at a wavelength of $632.5 \mathrm{~nm}$ is used as the light source. The laser light is focused on polarizer P1 for obtaining linearly polarized light. The circularly polarized light is generated, by inserting a quarter mica retardation plates behind the linear polarizer. The output polarized light is focus to soybean oil and again pass through linear polarizer, quarter wave plate and recorded on photodiode detector/CCD camera, which is controlled and operated with Lab software. The soybean oil is used as scattering phantom. 
The experimental setup for Mueller matrix polarimetry is shown schematically in Fig. 1. A light source with spot size is less than $2 \mathrm{~mm}$ passes through a Polarizer (P1) and quarter wave-plate (QWP1) and impinges on the sample. The scattered light which emerges from the sample passes through a analyzer (P2) and quarter wave-plate(QWP2), and is then recorded by the detector connected to lock in amplifier or CCD camera system (Pico Star, Lab Vision). The CCD resolution is 12 bit and the lab view software is used for data analysis. The scattering medium (soybean oil) is placed in a cylindrical thin-walled quartz cell $(3 \times 2$ $\mathrm{cm})$. Soybean oil is an inexpensive, non-toxic liquid with dielectric properties similar to very low-water-content fatty tissue. For this reason, it is used as the tissue phantom. [38]

\section{Experimental results}

In this study the soybean oil is used as tissue like phantom. We recovered optical information by selectively detecting a transmitted component of the scattered photon flux that has its initial polarization state preserved. These photons transmitted through or reemitted from a multiply scattering medium by using relatively inexpensive Mueller matrix polarimeter provides the basis for several potential applications. The measurement technique is based upon an operational principle, which involves the modulation of a polarization state. The resulting modulated light signal is collected by the detector/CCD camera and is analyzed pixel by pixel to calculate individual intensity patterns, which correspond respectively to the 16 components of the scattering Mueller matrix. In brief, the two polarizers and quarter-wave plates inserted in the probing and analyzing beam paths, are generate a periodic signal, this signal carries information about the properties of the medium which induces the transformation of the polarization state of the modulated probing light. The experimental procedure requires collecting of 16 intensity images at various orientations of the polarizing components. The described procedure provides the possibility to calculate the scattering Mueller matrix for a given sample.

The Stokes parameter I of the system in Fig.2 represents the magnitude of the intensity of the scattered light. Thus, any abrupt change in the detected signal indicates strong discontinuity in the refractive index of the specimen. Along with I, other Stokes parameters, $Q, U$ and $V$ can be used to detect structural changes that are not simply detected from I. Other Stokes parameter images of $U$ and $V$ show supplement information that there is no apparent level of stress inside the scatterer. Therefore, by analyzing the corresponding series of the polarization patterns one can trace scattering events of different order. Specifically, the data analysis of Fig. 3, suggests several interesting observations regarding the general properties of the scattering Mueller matrix. First, the magnitude of the off-diagonal components of the scattering Mueller matrix is significantly smaller than the magnitude of the diagonal components.

In the present experiments, we are able to trace the polarization patterns and to verify that the existing magnitude distributions are preserved. The magnitude and the sign of most of the spatial extent of the matrix components for Fig. 3, 4 closely resemble the form of the Mueller matrix for scattering medium. The next important observation is that the experimental results clearly display several symmetry properties of certain matrix components for homogeneous scattering medium. The seven out of sixteen are independent and other can be calculated through symmetry relation. [39] By comparing the images of Fig. 3, 4, and 5, one can identify the unique features of the Mueller matrix for scattering medium. The axial symmetry of the system provides relation between all the Mueller matrix 
components and reduces the number of measurements, which reduces the observation time. These symmetry relations describe that the seven independent elements are, [40]

$$
m_{11}(\theta, \varphi), m_{12}(\theta, \varphi), m_{14}(\theta), m_{22}(\theta, \varphi), m_{23}(\theta, \varphi), m_{24}(\theta, \varphi), m_{44}(\theta),
$$

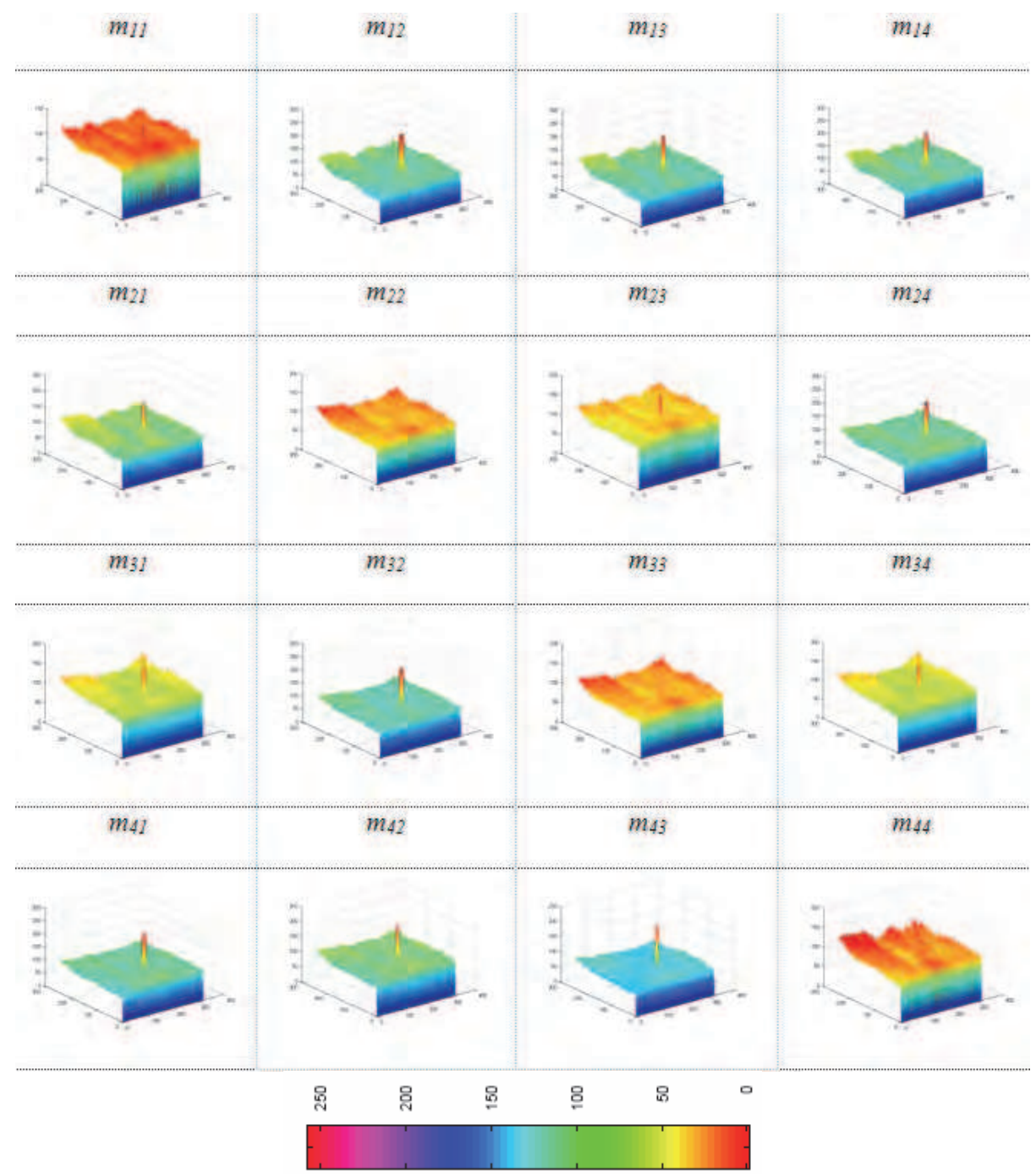

Fig. 2. Transmitted Mueller matrix components (3D) corresponding to a 16 images of scattering medium. The images are taken with the experimental setup in Fig.1. The scale bar is adjusted so that red represent the maximum irradiance, yellow to middle one, green for minimum irradiance and blue means "no light" or component change by an order of magnitude. All displayed images are $3 \times 3 \mathrm{~cm}$. 
$I$

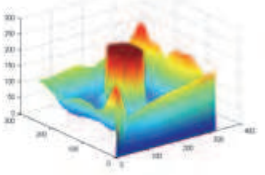

B

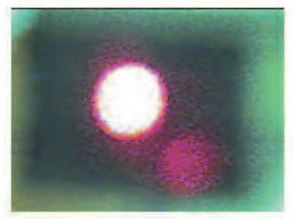

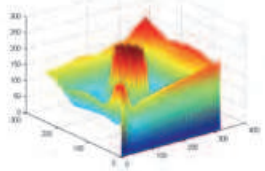

$Q$
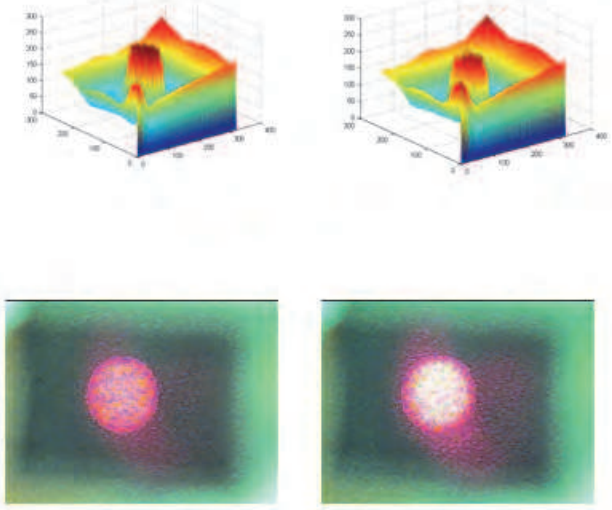

$U$

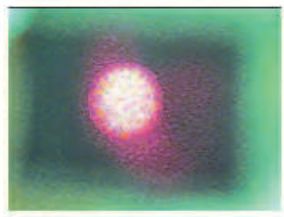

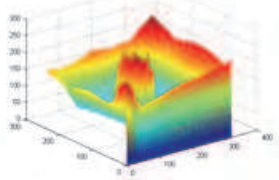

$V$

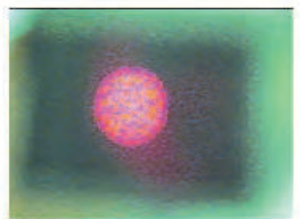

Fig. 3. the Stokes vector I, Q, U, and V are shown, I present the total irradiant power profile, $\mathrm{Q}$ for horizontal polarized light, $\mathrm{U}$ for $+45^{0}$ polarized incident light and $\mathrm{V}$ the right circular polarized light. (a) Represents the 3-D images and (b) represent the direct transmitted intensity for all the Stokes vectors.

Where $\theta$ is the rotation angle of the transmission axis of the polarizer, $\varphi$ is the phase shift of the retarder and the other nine dependent elements are:

$$
\begin{gathered}
m_{13}(\theta, \varphi)=m_{12}\left(\theta, \varphi+\frac{\pi}{4}\right), \\
m_{21}(\theta, \varphi)=m_{12}(\theta, \varphi) \\
m_{31}(\theta, \varphi)=-m_{13}(\theta, \varphi)=m_{12}\left(\theta, \varphi-\frac{\pi}{4}\right), \\
m_{32}(\theta, \varphi)=-m_{23}(\theta, \varphi)=m_{23}\left(\theta, \varphi \pm \frac{\pi}{4}\right), \\
m_{33}(\theta, \varphi)=-m_{22}\left(\theta, \varphi-\frac{\pi}{4}\right), \\
m_{34}(\theta, \varphi)=m_{24}\left(\theta, \varphi-\frac{\pi}{4}\right), \\
m_{41}(\theta, \varphi)=m_{41}(\theta)=m_{14}(\theta), \\
m_{42}(\theta, \varphi)=m_{24}(\theta, \varphi) \\
m_{43}(\theta, \varphi)=-m_{34}(\theta, \varphi)=m_{24}\left(\theta, \varphi+\frac{\pi}{4}\right)
\end{gathered}
$$

Fig. 6. displays the matrix array, which represents the detector reading specific to a linear polarizer lie in the first row, first column elements, those specific to a quarter wave plate lie in the fourth row, fourth column elements. 


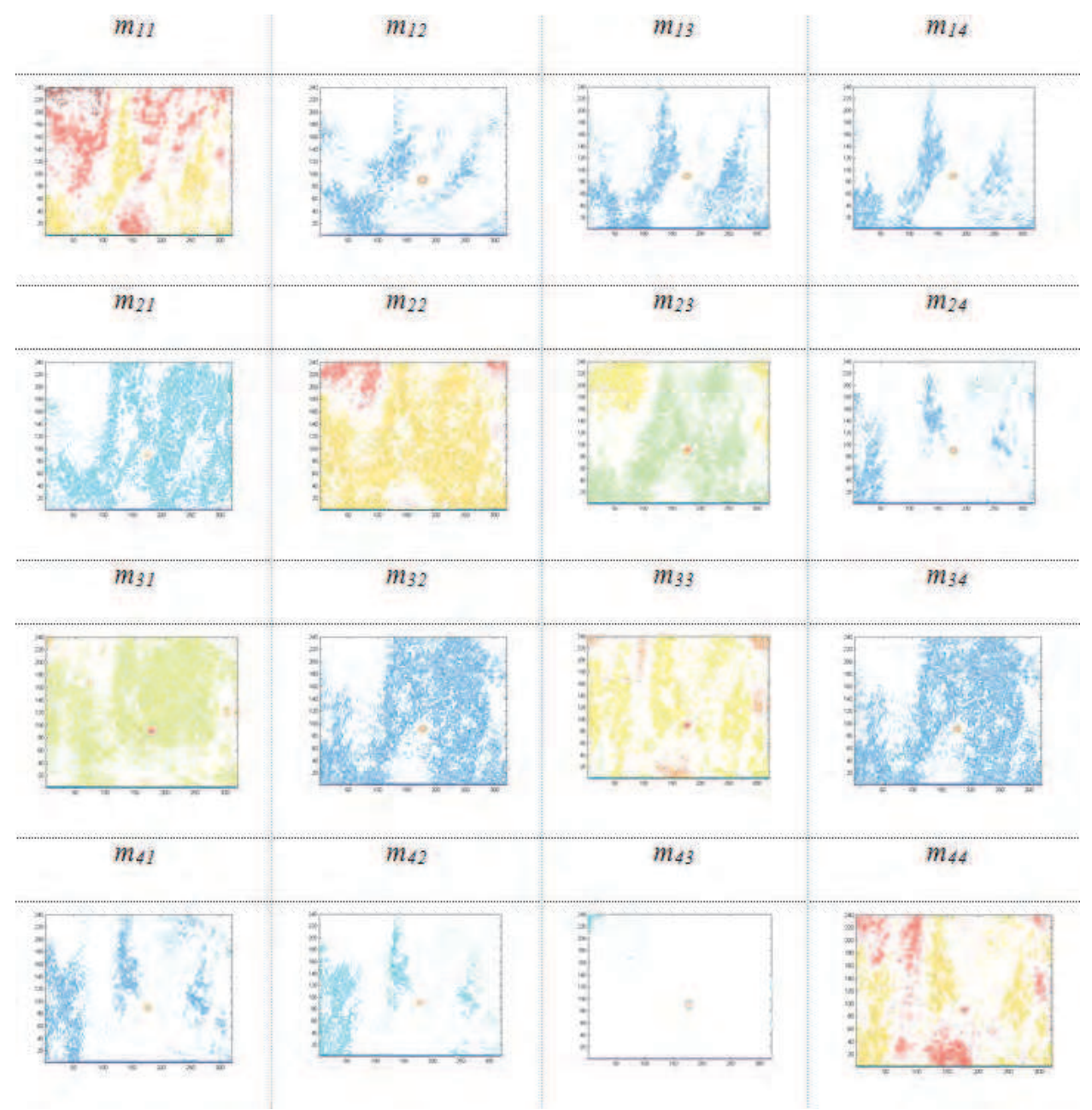

Fig. 4. Transmitted Mueller matrix components (2D) corresponding to a scattering. These 2D images are derived through 49 measurements of Mueller matrix polarimeter. The central spot presents the output transmitted irradiance by scatterer along with scattering pattern.

Making the above 49 measurements of polarized light from a scatterer will produce 16 matrix element pattern. Each one is an electric field dependent intensity measurement for a particular arrangement of input-output optics. These 16 curves contain all the information that can be learned from a scattering experiment. Choosing input-output optical combinations, different than described above, will produce a set of patterns drastically different in appearance but not fundamentally different in information content. When the 16 matrix elements are measured the data is ready for analysis. For certain perfect particles like spheres, fibers, and mixtures of perfect particles, the matrix elements can be exactly predicted. So the set of 16 measurements will stand as the signature of the scatterer as described by polarized scattered light. 


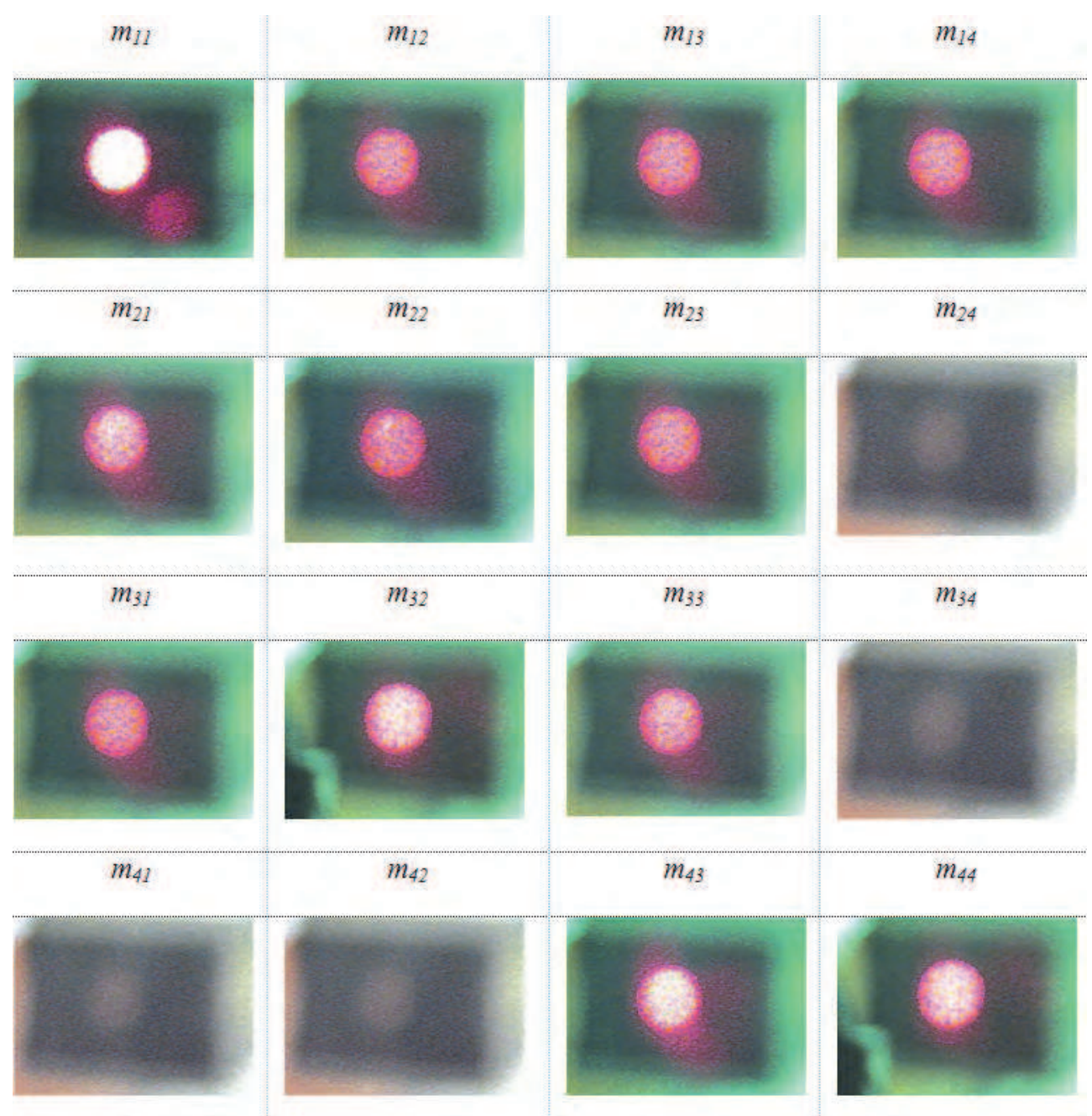

Fig. 5. Experimental transmitted polarization images of Mueller matrix components corresponding to a scattering medium of randomly distributed particles. The images corresponds the direct scatterer irradiance, measured through polarization discrimination technique.

Mueller matrix polarimetric pattern analysis predicts interesting information about the medium. The ballistic, snake and diffuse photons reaching the camera contributes to the formation of direct image. The diffuse photons have suffered multiple scattering before exiting the scattering medium. Ballistic photons completely preserve the polarization properties of the irradiant light after passing through scattering medium. The snake photons recorded by the detector are partially polarized but to the smaller degree then the ballistic photons, because snake photons partially preserve polarization. The diffuse photon depolarized for thick sample. The collective image of these three scattered photon provides 
useful information and characterizes the scattering medium in term of size shape under Raleigh and Mie scattering theory.

The diagonal elements of Mueller matrix consist on linear and circular polarization pattern. The Mueller matrix $m_{11}$ describe the properties of the total irradiance of light source and provides less information comparing to other elements of the matrix array, but all other elements are normalized through it. The $\mathrm{m}_{22}$ composed of linear horizontal and vertical polarization state. The liner polarization preserve through longer distance in the scattered as compared to circular light The concentration, size and shape of the particles in scattering medium can be predicted through careful analysis of this element alomgwith other linear elements of the matrix. If the value of this element is zero or below, then the medium obey Raleigh theory and the size of the particle is small as compared to the irradiated wavelength. If its value is greater than zero than it can be explain through Mie theory and particles of the scattering medium are larger in size. $m_{33}$ depend on $\pm 45^{0}$ linear polarization state and describes the properties almost close to $m_{22}$ element. The last element $m_{44}$ of the Mueller matrix compose on circular polarization. If the size of scatterer is larger, then the magnitude of this element will be in negative otherwise greater than or equal to zero. The difference between normal and malignant biological tissues can be characterized through this element. But for larger scattered concentration, it is less informatics because the data is taken through diffuse photon and the preservation of circular polarization is not dominant in this medium. If this element is measured through the ballistic and snake photons contribution then it reviles a significant role in characterization of biological tissues. In our case the experimental data shows decline in the diagonal elements from top to bottom that conforms the preservation of linear polarization in diffuse medium for longer distance compared to circular one. As $\mathrm{m}_{44}$ is greater than zero, which predicts that the size of scattered is larger than the irradiating wavelength. The size of the particle can be numerically calculated through Mie scattering theory. [41]

\begin{tabular}{|l|l|l|l|}
\hline 0.986 & 0.007 & 0.004 & 0.0003 \\
\hline 0.007 & 1.003 & -0.007 & 0.009 \\
\hline 0.008 & -0.007 & 0.992 & -0.003 \\
\hline 0.003 & -0.006 & -0.007 & 0.989 \\
\hline
\end{tabular}

a

\begin{tabular}{|l|l|l|l|}
\hline 0.910 & 0.657 & -0.314 & 0.097 \\
\hline 0.739 & 0.732 & 0.793 & 0.083 \\
\hline 0.435 & 0.243 & 0.620 & -0.213 \\
\hline 0.133 & 0.421 & 0.136 & 0.751 \\
\hline \multicolumn{4}{|c|}{ b } \\
\hline
\end{tabular}

Fig. 6. Mueller matrix data for transmitted polarized laser beam (a) no sample (air) and (b) for scattering turbid sample.

First row and first column of the Mueller matrix except $\mathrm{m}_{14}$ and $\mathrm{m}_{41}$ describe the linear polarization pattern. Each and every element of this group is very informatics, and describes the structure of the dense diffuse scattered. From Fig. 3, 4, and 5 we see that the intensity contrast reduces from right to left and top to bottom of the Mueller intensity matrices except $\mathrm{m}_{13}$, which tells about the enriched optical activity and highly birefringence of the sample. The higher value of element $m_{13}$ is due to randomization of the sample molecules, when the 
incident light is of $\pm 45^{0}$ polarized. From analysis of the elements of this group the normality and abnormality of the medium can easily be defined. In Fig.7 the depolarization of linearly polarized light through scattered is represented and it increases with the depth of soybean oil.

The elements in the middle of this group $\mathrm{m}_{22}, \mathrm{~m}_{23}, \mathrm{~m}_{32}$, and $\mathrm{m}_{33}$ can obtain through $\pm 45^{0}$ linear polarization. If some properties of the scattered cannot be obtain through the elements of other group they can be characterize through it. The elements $m_{23}$ and $m_{32}$ decline for dense scattering medium and the scattering angle for these elements is very small. The reduction of these elements directly related to the variation in structure of the scattered.

The last row and last column of this matrix set consist on circular polarization pattern. This group exhibits the depolarization properties of the medium. The depolarization is faster in dense as in case of circular one. The light is equally right and left-hand polarized and the effect is strongest in the center, near the laser entry point. Here the scattered light has undergone only a few scattering events and the polarization effects are strongest. With increasing distance from the point of light incident, the number of scattering events increases and eventually the polarization information is lost, the value of the $\mathrm{m}_{44}$ approaches zero. The majority of the elements of this group shows decline in the magnitude for dense medium and predict that the scattering cross-section of medium is small for this wavelength and the circular polarization preservation of light is weaker.

The next important observation is that the experimental results in Mueller matrix array display several symmetry properties and relations among them. This can be seen in Fig. 3-6 and these derived symmetry relations hold, of course, if the scattering medium contains one kind of randomly distributed asymmetrical particles or optically active. Some elements of the matrix have same behavior and other one are of same shape but rotated through $90^{\circ}$ as indicated in equation 8, 9 of this paper. All sixteen Mueller matrix components together provide a "finger print" of the scattering medium under investigation. As just shown, looking at the entire Mueller matrix often enables one to distinguish qualitatively between two media. Lot of information about particle size, refractive index, particle shape etc. has to be found in the Mueller matrix by careful analysis of the matrix elements. However, further information may be gained, for example, by measuring the diffuse backscattering and backreflectance at different incident and observation angles, or time-dependent polarization effects.

In this study we presented a polarization discrimination scattering experiment and have taken care to establish unambiguously the coordinate systems involved, the redundancy of certain measurements and the importance of particular orientations of optical element combinations. We believe that these concepts are important for understanding and fully appreciating optical polarization and that this approach is attractive because it discusses the inexpensive and non invasive procedures that are equally valid.

\section{Conclusion}

We describe the Mueller matrix polarization discrimination (MMPD) technique for characterization of highly scattering media(soybean oil) through laser beam. In our experiments, the scattering regime was adjusted to be at the incipient transition between single and multiple scattering. From an experimental standpoint the scattering is most challenging and on the other hand, it is rich in information content because the low-order scattering events are responsible for non-trivial polarization features. Our results 
demonstrate that the Mueller matrix components satisfy symmetry relations. These measurements provide detailed information about the changes in the magnitude and sign of Mueller matrix components. This should offer more insight and could lead to novel procedures for characterizing scattering phenomena. We discuss the entire experimental measured Mueller polarized matrix in detail for extracting the taking information about the structure, size, and shape of the scattering particles in term of its output polarization. This has the potential of characterization of turbid sample for their optical properties through polarized laser radiations.

We concluded that soybean oil is optical active, less retardence and highly depolarizing. All these characteristic describe soybean oil an accurate tissue like phantom with low absorption and higher scattering coefficient. Further study and characteristic of soybean oil can be deduced with application of Raleigh and Mie scattering models.

\section{References}

[1] A. Vitkin, R. D. Laszlo, and C. L. Whyman, "Effects of molecular asymmetry of optically active molecules on the polarization properties of multiply scattered light", Opt.Exp. 10, 222-229 (2002).

[2] S. Firdous, M. Fuzail, M. Atif, and M.Nawaz Polarimetric Characterization of Ultra-High Molecular Weight Polyethylene (UHMWPE) for Bone Substitute Biomaterials, Optik 122 (2) 99-104 (2011)

[3] S. Firdous, W. Waqas, M. Idrees,M. Atif, M. Nawaz, and M. Ikram, Polarization Sensitive Optical Imaging of Biomaterials using Mueller matrix Polarimetric Algorithm, Lasers in Medical Science (submitted 2011)

[4] R. R. Ansari, S. Bockle, and L. Rovati, "New optical scheme for a polarimetric- based glucose sensor", J. Biomed. Opt. 9, 103-115 (2004).

[5] D. Ge, Y. Cai, and Q. Lin, "Propagation of partially polarized light Gaussian Schellmodel beam in dispersive and absorbing media", Opt. Commun. 229, 93-98(2004).

[6] S. Firdous, K. Hassan, and M. Ikram, Formulation of Mueller Matrix and Modeling of depolarizing and scattering of nitrobenzene in a Kerr Cell, Appl. Opt. 44, 7,11711177 (2005)

[7] C. Cote, I. A. Vitkin, "Balanced detection for low noise precision polarimetric measurements of optically active multiply scateering tissue phantoms", J. Biomed. Opt. 9, 213-220 (2004).

[8] A. H. Hielscher, J. R. Mourant, and I. J. Bigio," Influence of particle size and concentration on the diffuse backscattering of polarized light from tissue phantoms and biological cell suspensions," Appl. Opt. 36, 125-135 (1997).

[9] P. Yang, H. Wei, G. W. Kittawar, Y. X. Hu, D. M. Winker, C. A. Hostetler, and B. A. Baum, "Sensitivity of the backscattering Mueller matrix to particle shape and thermodynamic phase", Appl. Opt. 42, 4389-4395 (2003).

[10] L. Jin, T. Hamada, Y. Otani, and N. Umeda, "Measurement of charactristic of magnatic fluid by the Mueller matrix imaging polarimeter", Opt. Eng. 43, 181-185(2004).

[11] J. T. Oh, S. W. Kim, "Polarization optical coherence tomography for photoelasiticty testing of glass/epoxy composites", Opt. Exp. 11, 1669-1676 (2003).

[12] S. L. Jacques, M. R. Ostermeyer, L. Wang, and D. V. Stephens, "Polarized light transmission through skin using video reflectometry: toward optical tomography of superficial tissue layers," Proc. SPIE 2671, 199-210 (1996). 
[13] M. C. Pierce, J. Strasswimmer, B. H. Park, and J. F. Deboer, “Birefrangence measurement in human skin using polarization-sensitive optical coherence tomography", J. Biomed. Opt. 9, 287-291 (2004).

[14] S. Jiao, G. Yao, and L.V.Wang, “ Depth-resolve two-diamentional Stokes vector backscattered light and Mueller matrix of bialogical tissues measured with optical coherence tomography", App. Opt. 39, 6318-6324 (2000).

[15] I. Berezhnyy, and A. Dogariu, "Time-resolved Mueller matrix imaging polarimetry" Opt.Exp. 12, 4635-4649 (2004).

[16] J. F. Deboer, T. E. Milner, "Review of polarization sensitive optical coherence tomography and stokes vector determination", J. Biomed. Opt. 7, 359-371 (2002).

[17] J. L. Pezzaniti, R. A. Chipman, "Mueller matrix imaging polarimetry", Opt. Eng. 34, 1558-1568 (1995).

[18] V. Sankarn, J. T. Walsh, and D. J. Maitland, "polarized light propagation through tissue phantom, ehms containing densely packed scatterers", Opt. Lett. 25, 239-241 (2000).

[19] R. Cross, B. Heffner, and P. Hernday, " Polarization measurement goes automatic", Lasers and Optronics, 10, 25-26 (1991).

[20] A. Nezhuvingal, Y. H. Anumula, and B. D. Cameron, "Mueller matrix optical imaging with application to tissue diagnistics", Proc. SPIE. 4961, 1-10 (2003).

[21] F. R Brehonnet, B. L. Jeune, P. Elies, J. Carious, and J. Lotrain, "Optical media and target charactrization by Mueller matrix decomposition", J. Phy. D. 29, 34-38 (1996).

[22] G. W. Kittawar, D. J. Gray, "Mueller matrix imaging of target in turbid media:effect of the volume scattering function", App. Opt. 42, 7225-7230 (2003).

[23] J. S. Baba, J. R .Chung, A. H. DeLaughter, B. D. Cameron, and G. L. Cote', "Development and calibration of an automated Mueller matrix polarization imaging system", J. Biomed. Opt. 7, 341-349 (2002).

[24] S. G. Demos and R. R. Alfano, "Optical polarization imaging," Appl. Opt. 36, 150-155 (1997).

[25] W. S. Bickel and W. M. Bailey, "Stokes vectors, Mueller matrices, and polarized scattering," Am. J. Phys. 53, 468-478 (1985).

[26] F. Delplancke, "Automated high-speed Mueller matrix scatterometer," Appl. Opt. 36, 5388-5395 (1997).

[27] W. S. Bickel, A.J. Watkins, and G.Videen," The light-scattering Mueller matrix elements for Rayleigh, Rayleigh-Gans, and Mie spheres," Am. J. Phys. 55, 559-561 (1987).

[28] E. Collett, Polarized Light: Fundamentals and Applications, Marcel Dekker, New York (1993).

[29] E. Hecht, Optics, 3rd ed., pp. 322-323, 366-367, 370, Addison Wesley Longman, Reading, MA (1998).

[30] C. Brosseau, Fundamentals of Polarized Light: a Statistical Optics Approach, Wiley, New York (1998).

[31] V. Sankarn, M. J. Everett, D. J. Maitland, and J. T. Walsh, “Compression of polarized light propagation in bialogical tissue and phantom", Opt. Lett. 24, 1044-1046 (1999).

[32] D. H. Goldstein, "Mueller matrix dual-rotating retarder polarimeter", Appl. Opt. 31, 6676-6683 (1992).

[33] D. S. Kilger, J. W. Lewis, and C. Einterz Randall, Polarized Light in Optics and Spectroscopy, Academic, San Diego, CA (1990). 
[34] S. Firdous and M. Ikram, Laser stokes polarimetry for the Characterization of biomaterials using liquid crystal variable retarders, Proc. SPIE 6632 (2007).

[35] E. A. West, M. H. Smith, "Polarization errors associated with birefrangent waveplates", Opt. Eng. 34, 1574-1580 (1995).

[36] D. H. Goldstein, R. A. Chipman, "Error analysis of a Mueller matrix polarimeter", J. Opt. Soc. Am. A. 7, 693-700 (1990).

[37] P. A. Williams, A. H. Rose, and C. M. Wang, "Rotating-polarizer polarimeter for accurate retardance measurement", Appl. Opt. 36, 6466-6472 (1997).

[38] S. T. Flock, S. L. Jacques, B. C. Wilson, W. M. Star, and M. V. Gemert, "Optical properties of intralipid: A phantom medium for light propagation studied", Laser Surg. Med.12, 510-519 (1992).

[39] M. j. Rakovic, G. W. Kittawar, M. Mehrubeoglu, B.D.Cameron, L. V. Wang, S.Resteger, and G.L.Cote., "Light backscattering polarization pattern from turbid media:theory and experiment",Appl. Opt.38, 3399-3408 (1999)

[40] S. Firdous, M. Ikram, " Charactrization of soybean oil through diffusely backscattered polarized light with matrix calculus-II",IEEE proceeding INCC 2004,115-123 (2004)

[41] H. C. Van de Hulst, Light Scattering by Small Particles, Dover, New York (1981). 


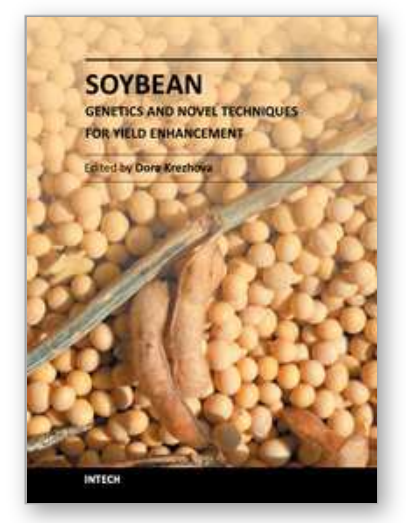

\author{
Soybean - Genetics and Novel Techniques for Yield Enhancement \\ Edited by Prof. Dora Krezhova
}

ISBN 978-953-307-721-5

Hard cover, 326 pages

Publisher InTech

Published online 07, November, 2011

Published in print edition November, 2011

This book presents the importance of applying of novel genetics and breading technologies. The efficient genotype selections and gene transformations provide for generation of new and improved soybean cultivars, resistant to disease and environmental stresses. The book introduces also a few recent modern techniques and technologies for detection of plant stress and characterization of biomaterials as well as for processing of soybean food and oil products.

\title{
How to reference
}

In order to correctly reference this scholarly work, feel free to copy and paste the following:

Shamaraz Firdous (2011). Polarization Sensitive Optical Imaging and Characterization of Soybean Using Stokes-Mueller Matrix Model, Soybean - Genetics and Novel Techniques for Yield Enhancement, Prof. Dora Krezhova (Ed.), ISBN: 978-953-307-721-5, InTech, Available from:

http://www.intechopen.com/books/soybean-genetics-and-novel-techniques-for-yield-

enhancement/polarization-sensitive-optical-imaging-and-characterization-of-soybean-using-stokes-muellermatrix-m

\section{INTECH}

open science | open minds

\section{InTech Europe}

University Campus STeP Ri Slavka Krautzeka 83/A 51000 Rijeka, Croatia Phone: +385 (51) 770447

Fax: +385 (51) 686166 www.intechopen.com

\section{InTech China}

Unit 405, Office Block, Hotel Equatorial Shanghai No.65, Yan An Road (West), Shanghai, 200040, China 中国上海市延安西路65号上海国际贵都大饭店办公楼405单元 Phone: +86-21-62489820

Fax: $+86-21-62489821$ 
(C) 2011 The Author(s). Licensee IntechOpen. This is an open access article distributed under the terms of the Creative Commons Attribution 3.0 License, which permits unrestricted use, distribution, and reproduction in any medium, provided the original work is properly cited. 